\title{
С.А. Пименов
}

\section{ФОРМУЛИРОВКА МЕТОДА ОЦЕНКИ ВЕРОЯТНОСТИ БЕЗОТКАЗНОЙ РАБОТЫ КОНСТРУКЦИЙ НА ОСНОВЕ СОЧЕТАНИЯ СТАТИСТИЧЕСКОГО МОДЕЛИРОВАНИЯ И ЧИСЛЕННЫХ МЕТОДОВ ОЦЕНКИ НАПРЯЖЕНИЙ}

\begin{abstract}
Рассматриваются методы оценки вероятности безотказной работы конструкций. Приведена формулировка метода оценки вероятности безотказной работы конструкций на основе сочетания статистического моделирования и численных методов оценки напряжений. В основе метода лежит модель «нагрузка - несущая способность», учитывающая: стохастичность механических свойств материалов конструкции; случайность геометрических характеристик; нагрузки вероятностного характера.
\end{abstract}

Ключевые слова: вероятность безотказной работы, нагрузка, несущая способность, статистическое моделирование.

\section{1. Введение}

Сложный характер взаимодействия различных составляющих конструкций с окружающей средой и между собой, случайная природа прочности материалов и условий эксплуатации, неточность сведений о характере нагружения и условий отказа элементов конструкции требуют в расчетах на прочность и долговечность применения вероятностных методов анализа и использования в качестве критериев показатели надежности. Таким образом, вероятностные аспекты проблем прочности рассматриваются теорией надежности.

На текущий момент достаточно хорошо разработаны методы и подходы к оценке вероятности безотказной работы или надежности конструкции на основе модели «нагрузка - несущая способность» [1-6]. В рамках указанных методов условие безотказной работы конструкции, или условие прочности, представляет неравенство между нагрузкой $Q$ и несущей способностью $R$. Ввиду того, что и нагрузка и несущая способность подвержены случайному разбросу с определенными параметрами рассеяния, условие прочности приобретает вероятностный смысл. Таким образом, вероятность отказа конструкции есть вероятность невыполнения условия прочности. Условие прочности записывается в виде

$$
\Psi=R-Q>0,
$$

где $\Psi$ - функция неразрушимости.

Аналитические методы и подходы предполагают знание законов распределения (математическое ожидание, дисперсия, коэффициент вариации) всех определяющих параметров, входящих в функции нагрузки $Q$ и несущей способности $R$, а также наличие аналитической зависимости определяющих параметров конструкции в виде функции

$$
Z=\Psi\left(a_{1}, a_{2}, \ldots . a_{i}, \ldots a_{n}\right) .
$$

Для ряда конструкций получение аналитического выражения, определяющего взаимосвязь между параметрами нагрузки $Q$ и несущей способностью $R$, вызывает 
затруднение. В этих случаях для оценки надежности (вероятности безотказной работы) прибегают к алгоритмам статистического моделирования и численным методам оценки напряжений, например методу конечных элементов [7-10].

Математическая модель, которая является описанием системы, функционирующей в условиях всякого рода случайных воздействий, называется стохастической моделью системы [1], где уравнение вида (2) может быть задано аналитически или в виде конечно-разностной или конечно-элементной схемы.

Задача исследования надежности системы - исследование вероятностных свойств $Z$, когда имеются вероятностные характеристики $a_{i}(i=1,2, \ldots, n)$. Так как полной вероятностной характеристикой случайной функции является ее функционал распределения, а случайная величина - ее функция распределения, то для решения сформулированной задачи необходимо уметь строить функционалы и функции распределения. Их определение с помощью аналитических методов представляет известные трудности.

Поставленную задачу можно решить следующим образом:

1) Сформировать вероятностные характеристики $Z$, определяющие надежность системы, а с помощью натурных испытаний, экспериментов (в том числе вычислительных) или теоретических исследований выяснить вид $\Psi$;

2) На множестве выборок реализаций случайных величин $a_{i}$ реализовать алгоритм распределения параметров системы;

3) На совокупности реализаций модели решений $\Psi$ построить статистические оценки вероятностных характеристик $Z$.

Основная идея метода статистического моделирования (статистических испытаний - метод Монте-Карло) состоит в том, что многократно воспроизводится схема, являющаяся формальным математическим описанием процесса функционирования реальной системы и в то же время выступающая в качестве математической модели, вероятностные характеристики которой адекватны решениям задач математического анализа.

Теоретической основой метода статистического моделирования является широко известный в теории вероятностей закон больших чисел, устанавливающий, при определенных условиях, предельное равенство среднего арифметического случайных величин при бесконечном увеличении числа опытов.

Метод статистического моделирования является универсальным методом нахождения закона распределения $f(z)$ по известным законам распределения определяющих параметров $a_{i}$. Метод пригоден практически для любых математических моделей.

Следует заметить, что совместное использование модели «нагрузка - несущая способность», метода статистического моделирования и конечно-элементного анализа также широко освещено в литературе [3-6].

\section{2. Классический подход}

Общий алгоритм статистического моделирования для оценки вероятности безотказной работы на основе модели «нагрузка - несущая способность» представлен на рис. 1. При этом вычисление напряжений может производиться либо по аналитической функции $\Psi$, определяющей взаимосвязь между параметрами нагрузки $Q$ и несущей способности $R$, либо на основе расчета конечно-элементной модели (КЭМ). Это так называемый классический подход статистического моделирования. 


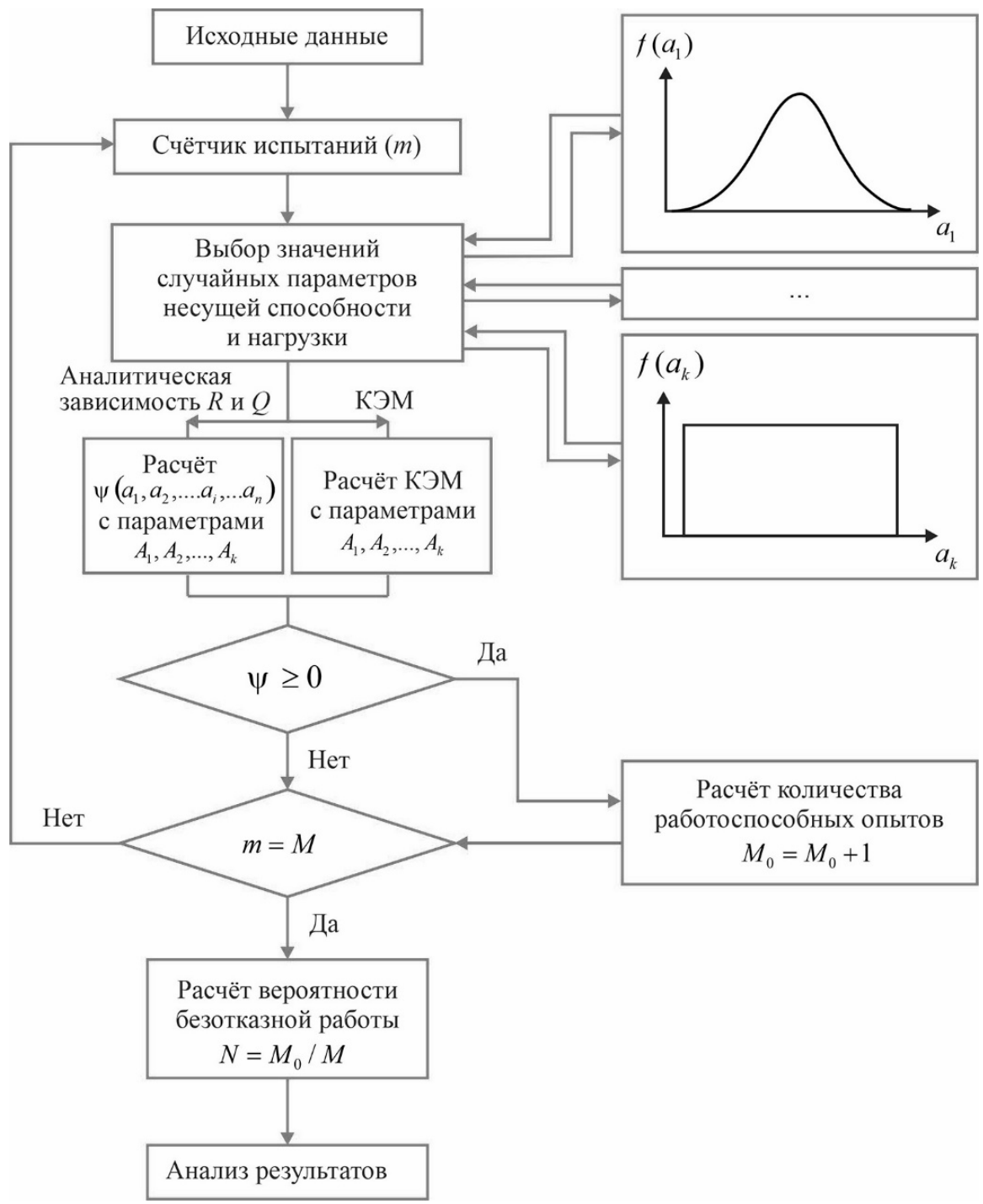

Рис. 1. Общий алгоритм статистического моделирования

Fig. 1. Statistical modeling algorithm

Одной из программных реализаций представленного подхода (рис. 1) является алгоритм модуля Probabilistic Design Analysis пакета программ ANSYS. В рамках данного модуля при решении задач оценки вероятности безотказной работы требуются следующие исходные данные: модельный файл, содержащий расчетную модель для вероятностного анализа (т.е. параметрическая КЭМ с фиксированным числом определяющих параметров как случайных величин) и собственно переменные (случайные величины), для которых либо должен быть задан закон распределения как набор констант (например, для нормального закона распределения следует задать математическое ожидание и дисперсию), либо должны быть заданы вариационные ряды с высоким объемом выборки. 
В классическом подходе можно выделить следующие не совсем корректные моменты:

1) В интерфейсе модуля Probabilistic Design Analysis, так же как и в других реализациях классического подхода, предлагается ограниченное число видов законов распределения. Что делать если случайный параметр имеет какой-то другой закон распределения, не описанный в интерфейсе? Этот параметр, конечно же, можно задать в виде вариационного ряда с высоким объемом выборки. Но при этом объем этой выборки должен быть сопоставим с объемами выборок сгенерированных данных для других случайных параметров, с заданными в интерфейсе законами распределения, что на практике не всегда возможно выполнить.

2) Если для какого-либо случайного параметра задан закон распределения, то генерация значений этого параметра выполняется с применением алгоритмов случайной генерации чисел, а получившийся, сгенерированный, вариационный ряд должен удовлетворять заданному закону распределения. При этом, при повторном запуске алгоритма генерации, можно получить совершенно другой вариационный ряд, но тоже удовлетворяющий заданному закону распределения. Никаких проверок, что эти ряды из одной и той же генеральной совокупности обычно не делается. И эти ряды никакого отношения к реальным конструктивным значениям не имеют, это абстрактные данные, а не данные, снятые средствами мониторинга конструкции.

3) Если случайные параметры заданы в виде вариационных рядов, а вариационные ряды - это результаты статистики мониторинга конструкции, то объем выборок этих рядов должен быть значительным (более 500 измерений, как показывает практика). Применение в классическом подходе вариационных рядов с низким объемов выборки может привести к некорректным результатам.

\section{3. Новая формулировка}

В отличие от классических реализаций предлагается новый подход, в котором в качестве исходных данных при решении задач оценки вероятности безотказной работы требуются: параметрическая КЭМ (как и в классической реализации) либо аналитическая функция $\Psi$, определяющая взаимосвязь между параметрами нагрузки $Q$ и несущей способности $R$; определяющие параметры как случайные величины, но заданные в виде вариационных рядов как результат статистики подобных конструкций. При этом объем выборки определяющих параметров может быть незначительным, а законы распределения не определены (т.е. имеет место эмпирические распределения определяющих параметров, без привязки к каким либо известным законам распределения).

Основой предлагаемой формулировки является алгоритм, в рамках которого следует многократно запустить расчет КЭМ конструкции для вычисления напряжений (либо вычислить напряжения на основе аналитической функции) с учетом данных вариационного ряда каждого из определяющих параметров. Алгоритм представляет собой вложенный цикл по вариационным рядам определяющих параметров (рис. 2). В теле цикла производится расчет КЭМ (или вычисляются напряжения на основе аналитической функции в слабейшем звене конструкции) с текущими значениями параметров из соответствующего вариационного ряда. Реализация такого алгоритма обычно производится языком команд системы инженерного анализа [4]. С использованием команд системы составляется управляющая программа для многократного запуска расчетного процесса. В результате расчета получается ряд чисел $\Psi_{i j}=R_{j}-Q_{i j}$, которые определяют кривую распреде- 
ления функции $\Psi$ для узлов КЭМ (или слабейшего звена). Здесь $Q_{i j}-$ уровень эквивалентных напряжений на $i$-й итерации для $j$-гоузла КЭМ, $R_{j}$ - уровень предельных напряжений для конструкции (в районе $j$-го узла КЭМ). Таким образом, в результате итерационного процесса $R_{j}, Q_{i j}$ представляются как эмпирические распределения для каждого узла КЭМ.

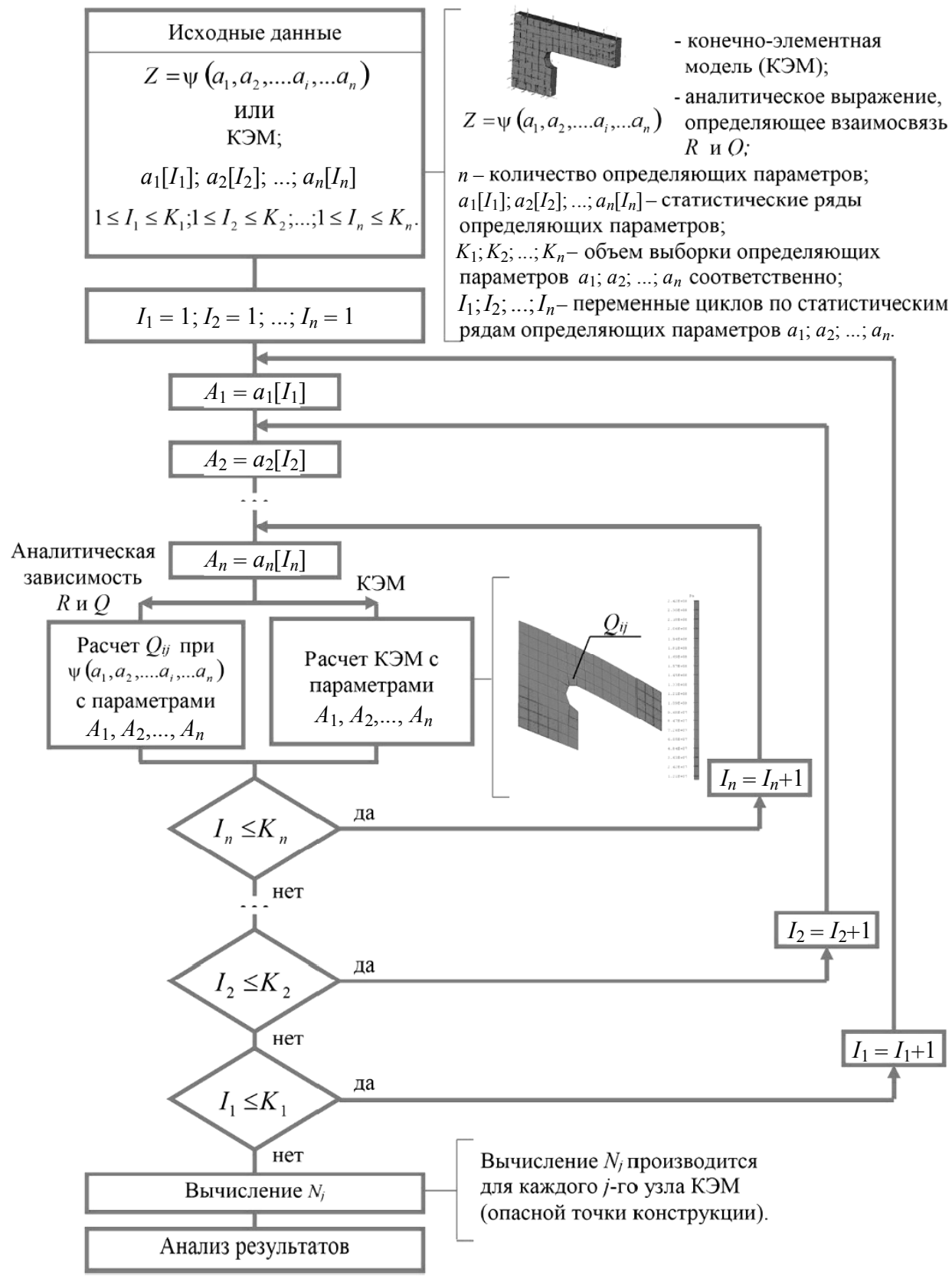

Рис. 2. Модифицированный алгоритм статистического моделирования

Fig. 2. A modified algorithm for statistical modeling 
Дальнейший расчет производится для опасного узла, исходя из принципа слабейшего звена, по методу определения вероятности безотказной работы (надежности), а именно выполнению условия неразрушимости по результатам расчета напряжений при эмпирических распределениях $R$ и $Q$ [2]. Данный метод выбран исходя из того, что нет оснований для принятия допущения о каком-либо конкретном распределении нагрузки $Q$ и несущей способности $R$, однако имеется достаточный объем эмпирических данных. В нашем случае в качестве эмпирических данных выступают данные вычислительного эксперимента - результаты расчета КЭМ (или вычисленные напряжения на основе аналитической функции в слабейшем звене конструкции) в теле цикла (рис. 2). Вводя обозначения $G_{j}=1-F_{R}\left(R_{j}\right)$ и $H_{j}=F_{Q}\left(Q_{i j}\right)$, запишем выражение для вероятности безотказной работы конструкции или надежности:

$$
N_{j}=\int_{0}^{1} G_{j} d H_{j} .
$$

Выражение (3) показывает, что вероятность безотказной работы (надежность) $N_{j}$ численно равна площади под кривой зависимости $G_{j}$ и $H_{j}[2]$. Используя результаты расчета КЭМ и соответственно данные о несущей способности $R_{j}$ и нагрузке $Q_{i j}$ объекта, строятся эмпирические функции распределения $F_{R}\left(R_{j}\right), F_{Q}\left(Q_{i j}\right)$ и, следовательно, $G_{j}$ и $H_{j}$. Площадь под кривой зависимости $G_{j}$ и $H_{j}$ определяется путем численного интегрирования. В результате расчета по каждому узлу КЭМ получается диаграмма изменения вероятности безотказной работы (надежности) по элементам конструкции.

Приведенный новый алгоритм (рис. 2) в общем случае представляет собой блок-схему из $n$ вложенных циклов. Количество определяющих параметров $n$ зависит от конструкции. Для выполнения расчета с произвольным значением $n$ алгоритм должен быть преобразован. Реализация такого алгоритма на ЭВМ осуществлена с применением рекурсии [4].

Основное отличие алгоритма (рис. 2) от классических алгоритмов статистического моделирования (рис. 1, как пример алгоритма модуля Probabilistic Design Analysis пакета программ ANSYS) - это применение формализованного метода определения вероятности безотказной работы при эмпирических распределениях $R$ и $Q$ (вычисление $N_{j}$ ). Изначально это графический метод вычисления показателей надежности. В рамках формализации в первую очередь производится выравнивание статистических распределений нагрузки и несущей способности с помощью некоторых аппроксимирующих кривых. Для построения функции распределения нагрузки по результатам решения задачи по всем итерациям и построения функции распределения несущей способности по указанному вариационному ряду производятся следующие действия:

- расчет области определения функций;

- построение графиков эмпирических функций распределения по результатам расчетов;

- аппроксимация результатов расчетов по методу наименьших квадратов с автоматическим выбором степени полинома;

- вычисление набора значений аппроксимации функции распределения на значениях исходного ряда;

- аппроксимация функции распределения с помощью сплайнов третьего порядка с асимптотическими граничными условиями. 
Область определения функций либо задается пользователем указанием минимального и максимального значения аргумента, либо задается с помощью расчета доверительного интервала математического ожидания по входному ряду с учетом заданного пользователем коэффициента доверительной вероятности. Расчет интервала выполняется с учетом распределения Стьюдента и оценки среднеквадратичного отклонения:

$$
\bar{x}-\frac{S_{1}}{\sqrt{n}} t_{\alpha, k}<I_{x}<\bar{x}+\frac{S_{1}}{\sqrt{n}} t_{\alpha, k},
$$

где $\bar{x}$ - среднее значение; $n$ - объем выборки (число измерений случайной величины $x$ ); $S_{1}$ - значение среднеквадратического отклонения с учетом коэффициента $K_{0}$, который учитывает смещенность оценки среднеквадратического отклонения при малых объемах выборки; $t_{\alpha, k}-$ значение квантили распределения Стьюдента для числа степеней свободы $K=n-1$ с доверительной вероятностью $P=1-\alpha / 2$.

Графики эмпирических функций распределения по результатам расчетов строятся как кусочно-постоянная функция, дополнительно строится кусочно-линейная интерполяция (рис. 3).

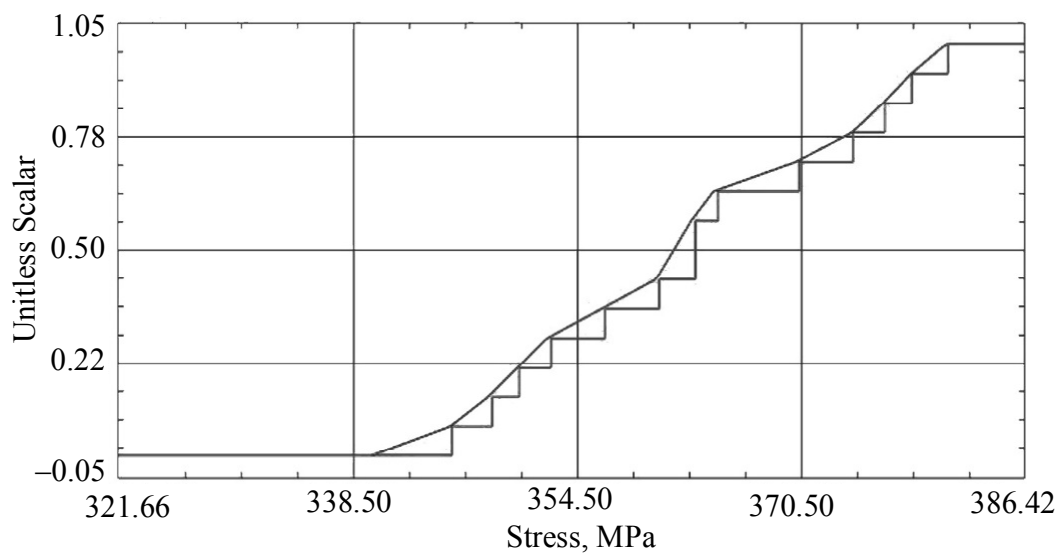

Рис. 3. Кусочно-линейная интерполяция

Fig. 3. Piecewise linear interpolation

Выравнивание статистических распределений осуществляется путем полиномиальной аппроксимации результатов расчетов по методу наименьших квадратов с автоматическим выбором степени полинома (рис. 4).

Полиномиальная аппроксимация обеспечивает нахождение коэффициентов полинома

$$
y(x)=a_{0}+a_{1} x+a_{2} x^{2}+\ldots+a_{m} x^{m}
$$

из решения следующей системы уравнений:

$$
\begin{aligned}
& c_{o} a_{o}+c_{1} a_{1}+c_{2} a_{2}+\ldots+c_{m} a_{m}=d_{0}, \\
& c_{1} a_{o}+c_{2} a_{1}+c_{3} a_{2}+\ldots+c_{m+1} a_{m}=d_{1}, \\
& \ldots \\
& c_{m} a_{o}+c_{m+1} a_{1}+c_{m+2} a_{2}+\ldots+c_{2 m} a_{m}=d_{m},
\end{aligned}
$$




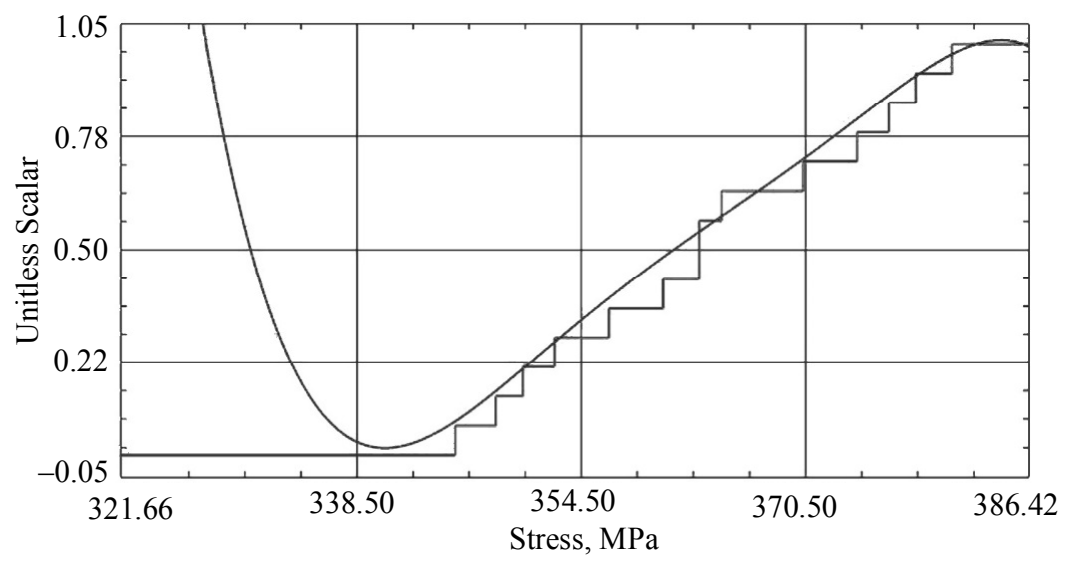

Рис. 4. Полиномиальная аппроксимация

Fig. 4. Polynomial approximation

где

$$
\begin{gathered}
c_{j}=\sum_{i=1}^{N_{x y}} x j, j=0,1,2, \ldots, 2 m ; \\
d_{k}=\sum_{i=1}^{N_{x y}} x_{i}^{k} y_{i}, k=0,1,2, \ldots, m .
\end{gathered}
$$

Полином (4) степени $m<N_{x y}$, где $N_{x y}$ - число пар $x_{i}$ и $y_{i}$, обеспечивает аппроксимацию (и интерполяцию) таблично заданной функции $y_{i}\left(x_{i}\right)$ с минимальной среднеквадратичной погрешностью:

где

$$
\begin{gathered}
E=K_{0} \sqrt{\sum_{i=1}^{N_{x y}} \varepsilon_{i}^{2} /\left(N_{x y}-1\right),} \\
\varepsilon_{i}=\left|\frac{y\left(x_{i}\right)-y_{i}\left(x_{i}\right)}{y_{i}\left(x_{i}\right)}\right|-\left|\frac{y\left(x_{i}\right)-y_{i}\left(x_{i}\right)}{y_{i}\left(x_{i}\right)}\right| / N_{x y} ;
\end{gathered}
$$

$K_{0}$ - поправочный коэффициент, учитывающий смещенность оценки среднеквадратического отклонения в зависимости от $N_{x y}$.

Если $m=N_{x y}$, то имеет место обычная интерполяция, в этом случае значения $y(x)$ при $x=x_{i}$ точно совпадают с заданными $y_{i}$. При $m<N_{x y}$ такого совпадения в общем случае нет, аппроксимация $y_{i}\left(x_{i}\right)$ по методу наименьших квадратов, таким образом, имеет более универсальных характер, чем обычная интерполяция.

Если достаточно ограничиться вычислением лишь коэффициентов $a_{0}, a_{1}, \ldots, a_{m}$ полинома (4) без вычисления значения погрешности $E$, то нет необходимости в хранении массивов $x_{i}$ и $y_{i}$. При этом достаточно вычислить $c_{j}$ (массив из $2 m$ чисел) и $d_{k}$ (массив из $m$ чисел) с помощью (6), (7) соответственно и далее решить полученную систему уравнений методом Гаусса.

Полиномиальная аппроксимация с автоматическим выбором степени полинома выполняется по следующему алгоритму:

1) Задается степень $m=1$ (линейная интерполяция), при этом массивы $x_{i}$ и $y_{i}$ сохраняются; 
2) После вычисления коэффициентов $a_{o}, a_{1}, \ldots, a_{m}$ методом Гаусса с помощью (8) вычисляется среднеквадратическая погрешность $E$ и сравнивается с заданной $E_{1}$;

3) Если $E>E_{1}$, то степень полинома $m$ увеличивается на 1 и так далее производится итерационный расчет, начиная с второго пункта перечисления настоящего списка;

4) Расчет прекращается, как только достигается $E<E_{1}$.

Примечание. Таким образом, производится полиномиальная аппроксимация функций распределения нагрузки $F_{Q}\left(Q_{i}\right)$ и несущей способности $F_{R}\left(R_{i}\right)$ исходя из результатов расчетов (вариационных рядов нагрузки $Q_{i}$ в контрольных узлах КЭМ) и данных о несущей способности конструкции (вариационный ряд $R_{i}$ ). Полиномиальная аппроксимация производится методом наименьших квадратов с автоматическим выбором степени полинома на основе погрешности, указанной на входе алгоритма.

По результатам аппроксимации методом наименьших квадратов производится вычисление значений функции распределения на значениях исходного ряда. Эти значения становятся исходными для расчетов, выполняемых на следующем шаге.

Метод наименьших квадратов достигает точности на значениях исходного ряда, но обладает существенными недостатками - большой погрешностью интерполяции при больших промежутках в значениях исходного ряда и погрешностью экстраполяции (рис. 4). Поэтому для экстраполяции на функции распределения на участках справа и слева от исходного ряда требуется введение сплайнов третьего порядка с асимптотическими граничными условиями.

Аппроксимация функции распределения с помощью сплайнов третьего порядка с асимптотическими граничными условиями выполняется с учетом гладкого (равенство первых производных) соединения участков функции между исходными значениями. Функция распределения асимптотически приближается к нулю слева и к единице справа, поэтому принимается, что в граничных точках определения первая производная равна нулю. Экстраполяция слева и справа области определения выполняется линейными участками с сохранением наклона (значения первой производной) на границах области определения (рис. 5).

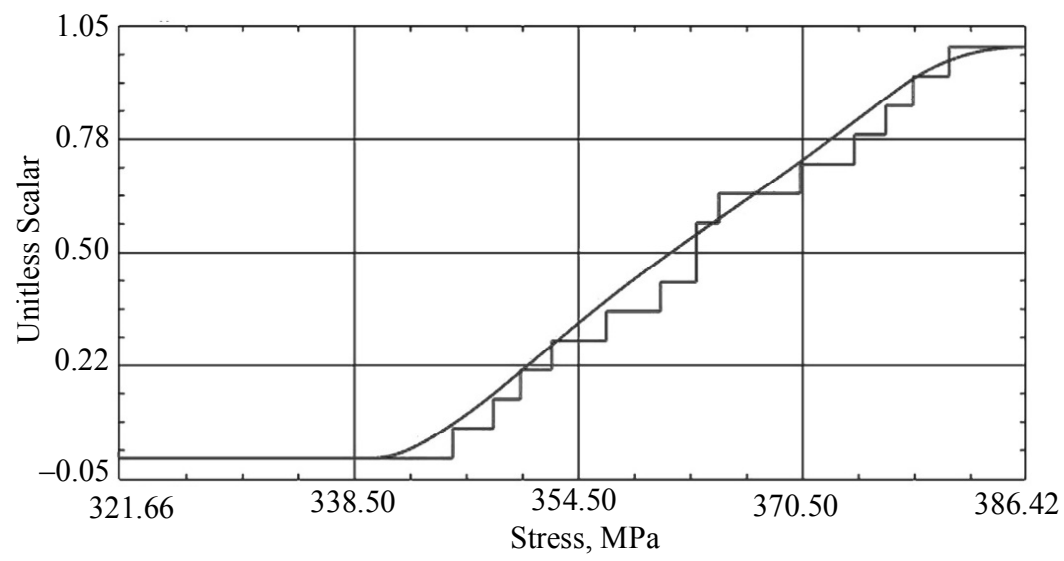

Рис. 5. Экстраполяция на границах области определения

Fig. 5. Extrapolation at the boundaries of a definition domain 
Из ранее приведенного видно, что производится выравнивание статистических распределений нагрузки и несущей способности с помощью некоторых теоретических кривых. Между теоретической кривой (как бы она хорошо подобрана не была) и статистическим распределением неизбежны некоторые расхождения. Возникает вопрос: объясняются ли эти расхождения только случайными обстоятельствами, связанными с ограниченным числом наблюдений, или они являются существенными и связаны с тем, что подобранная кривая плохо выравнивает данное статистическое распределение. Для ответа на этот вопрос служат критерии согласия.

В данном случае проверка расхождения между теоретическим и статистическим распределениями осуществляется на основе критериев согласия Колмогорова и Пирсона. По данным критериям проверяется гипотеза $H$, состоящая в том, что случайная величина $X$ подчиняется некоторому определенному закону распределения, полученному на основании заданного статистического материала (опытные или расчетные данные).

Схема применения критерия Колмогорова следующая:

1) Строятся статистическая функция распределения $F_{n}^{*}(x)$ и предполагаемая функция теоретического распределения $F(x)$ на основе ранее приведенной технологии выравнивания с помощью аппроксимационных функций (рис. 6);

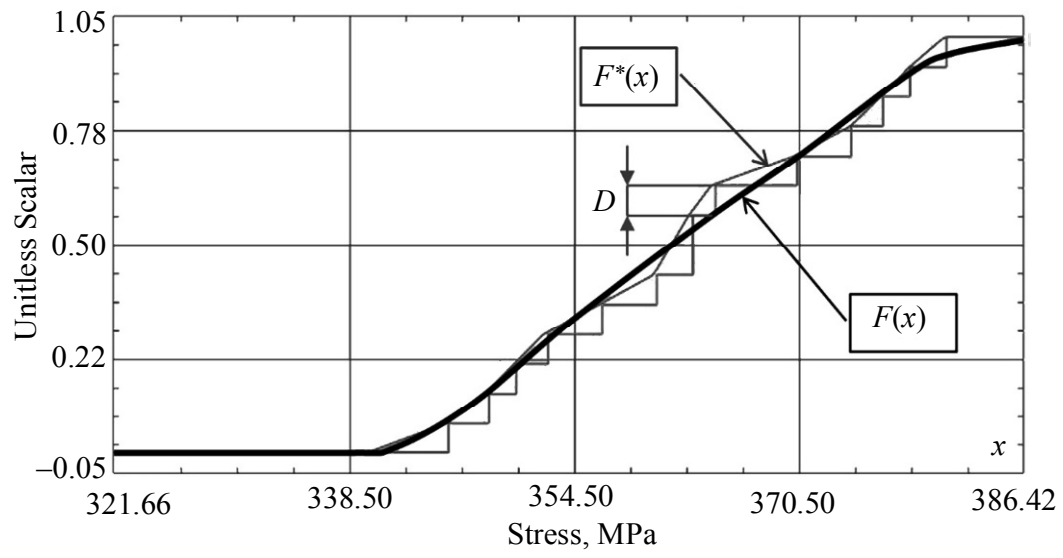

Рис. 6. Схема применения критерия Колмогорова

Fig. 6. Scheme for applying the Kolmogorov criterion

2) Определяется максимум $D_{n}$ модуля разности между этими распределениями:

$$
D_{n}=\max \left|F_{n}^{*}(x)-F(x)\right|
$$

3) Определяется величина $\lambda=D_{n} \sqrt{n}$ и по табл. 1 находится вероятность $P(\lambda)$;

Примечание. Величина $P(\lambda)$ есть вероятность того, что за счет чисто случайных причин максимальное расхождение между $F(x)$ и $F_{n}^{*}(x)$ будет не меньше, чем фактически наблюдаемое. 


\begin{tabular}{|c|c|c|c|c|c|c|c|}
\hline$\lambda$ & $P(\lambda)$ & $\lambda$ & $P(\lambda)$ & $\lambda$ & $P(\lambda)$ & $\lambda$ & $P(\lambda)$ \\
\hline 0.0 & 1.000 & 0.6 & 0.864 & 1.1 & 0.178 & 1.6 & 0.012 \\
\hline 0.1 & 1.000 & 0.7 & 0.711 & 1.2 & 0.112 & 1.7 & 0.006 \\
\hline 0.2 & 1.000 & 0.8 & 0.544 & 1.3 & 0.068 & 1.8 & 0.003 \\
\hline 0.3 & 1.000 & 0.9 & 0.393 & 1.4 & 0.040 & 1.9 & 0.002 \\
\hline 0.4 & 0.997 & 1.0 & 0.270 & 1.5 & 0.022 & 2.0 & 0.001 \\
\hline 0.5 & 0.964 & & & & & & \\
\hline
\end{tabular}

4) Если вероятность $P(\lambda)$ весьма мала, то гипотезу $H$ следует отвергнуть как неправдоподобную, при сравнительно больших $P(\lambda)$ её можно считать совместимой с опытными (расчетными) данными.

Схема применения критерия Пирсона:

1) Определяется мера расхождения $\chi^{2}$ по формуле

$$
W=\chi^{2}=k \sum_{i=1}^{n} \frac{\left(P_{i}^{*}-P_{i}\right)^{2}}{P_{i}}=\sum_{i=1}^{n} \frac{\left(m_{i}-k P_{i}\right)^{2}}{k P_{i}},
$$

где $P_{i}^{*}=m_{i} / k ; m_{i}$ - число значений в $i$-м разряде; $n$ - число разрядов;

2) Определяется число степеней свободы $K$ по формуле $K=n-s$ (число разрядов $n$ минус число независимых условий, связей $s$, наложенных на частоты $P_{i}^{*}$ );

3) По $K$ и $\chi^{2}$ с помощью таблиц распределения Пирсона находят вероятность того, что величина, имеющая распределение $\chi^{2}$ с $K$ степенями свободы, превзойдет данное значение $\chi^{2}$;

4) Если эта вероятность весьма мала (меньше 0.1), гипотеза $H$ отбрасывается как неправдоподобная, а если вероятность относительно велика, гипотезу $H$ можно признать не противоречащей опытным (расчетным) данным.

Основная область применения предлагаемого метода - оценка вероятности безотказной работы конструкций, нагруженных стационарной или квазистационарной случайной нагрузкой при следующих условиях:

1) Предполагается, что вид законов распределения нагрузки и несущей способности во времени не меняется. Неизменными также считаются и параметры законов распределения;

2) Для исследуемой конструкции, для ее опасного места, выведено аналитическое выражение $\psi\left(a_{1}, a_{2}, \ldots, a_{i}, \ldots, a_{n}\right)$, определяющее взаимосвязь $R$ и $Q$, либо конструкция задана в виде параметрической КЭМ с заданным числом определяющих параметров $a_{1}, a_{2}, \ldots, a_{i}, \ldots, a_{n}$;

3) Определяющие параметры конструкции $a_{i}$ полагаются независимыми случайными величинами, имеют произвольный закон распределения или заданы эмпирически в виде вариационных рядов.

Дополнительная область применения - оценка вероятности безотказной работы при случайных динамических нагрузках, представленных в виде детерминированных функций, зависящих от конечного числа случайных величин. Например: ударные воздействия, форма импульса которых неизменна, а амплитуда и длительность представлены как независимые случайные величины; синусоидальная вибрация, где амплитуда и частота - независимые случайные величины. В рамках предлагаемого метода возможно решить классическую задачу статистической ме- 
ханики с точки зрения теории надежности, т.е. вычислить вероятность безотказной работы с учетом случайного нагружения (например, заданного в виде широкополосной случайной вибрации) и случайных факторов на уровне геометрии и свойств материалов конструкции.

В предлагаемом методе случайными факторами могут быть: механические свойства материалов конструкции, геометрические характеристики конструкции и дефектов, условия нагружения.

\section{4. Заключение}

Разработанный метод предназначен для анализа вероятности безотказной работы при случайном нагружении. Его основной отличительной чертой является возможность использования в качестве исходных данных для решения задач оценки вероятности безотказной работы (надежности) определяющих параметров, заданных в виде вариационных рядов. Достоверность результатов достигается при незначительных объемах выборки, при этом закон распределения может оставаться неизвестным.

Основное преимущество - универсальность метода. С применением данного метода возможно проводить расчеты для широкого ряда конструкций с учетом статистического характера исходных данных. Ввиду возможности использования выборок малых объемов, применение метода актуально для конструкций, диагностика и систематический мониторинг которых затруднены вследствие конструктивных особенностей и специфики эксплуатации.

\section{ЛИТЕРАТУРА}

1. Волков В.М. Надежность машин и тонкостенных конструкций: учеб. пособие. Н. Новгород: Изд-во НГТУ, 2011. 365 с.

2. Капур К., Ламберсон Л. Надежность и проектирование систем. М.: Мир, 1980. 604 с.

3. Пименов С.A. Применение численных методов для оценки надежности конструкций // Новые промышленные технологии. 2010. № 3. С. 55-57.

4. Пименов С.А., Палкин И.Ю. Применение рекурсивных алгоритмов при оценке надежности конструкций // Наукоемкие технологии. 2011. № 4. С. 39-43.

5. Острейковский В.A. Теория надёжности. М.: Высшая школа, 2003. 464 с.

6. Probabilistic Design Methodology for Composite Aircraft Structures, National Technical Information Service (NTIS), Springfield, Virginia 22161, June 1999. URL: http://www.tc.faa. gov/its/worldpac/techrpt/ar99-2.pdf

7. Зенкевич О.К. Метод конечных элементов в технике. М.: Мир, 1975. $541 \mathrm{c.}$

8. NX Nastran Numerical Methods User's Guide. UGS, 2005. URL: http://docs.plm.automation. siemens.com/data_services/resources/nxnastran/10/help/en_US/custom/numerical/numerical.pdf

9. ANSYS Mechanical APDL Basic Analysis Guide, Release 15.0. ANSYS. Inc, November, 2013. URL: https://docplayer.net/45466427-Ansys-mechanical-apdl-basicanalysis-guide.html

10. Abaqus Analysis User's Manual, Release 6.1. Dessault Systems, 2010. URL: https://www. academia.edu/24831145/Abaqus_Analysis_Users_Manual

Статья поступила 17.02.2019

Pimenov S.A. (2021) FORMULATION OF A METHOD OF RELIABILITY ASSESSMENT FOR CONSTRUCTIONS ON THE BASIS OF A COMBINATION OF STATISTICAL MODELING AND NUMERICAL METHODS OF STRESS EVALUATION. Vestnik Tomskogo gosudarstvennogo universiteta. Matematika i mekhanika [Tomsk State University Journal of Mathematics and Mechanics]. 70. pp. 51-63

DOI $10.17223 / 19988621 / 70 / 5$ 
Keywords: reliability, load, bearing strength, statistical modeling.

In this paper, methods for estimating the reliability of constructions are considered. The formulation of a method for estimating the reliability of the design based on a combination of statistical modeling and numerical methods for evaluating stresses is given. The method is based on a "load - bearing capacity" model, which takes into account both stochasticity of mechanical properties of construction materials, randomness of geometric characteristics, and loads of a probabilistic nature.

This new method is a system of $n$ nested cycles. The number of governing parameters $n$ depends on the design. The load variation series is calculated in the body of the internal cycle. The load is calculated numerically in terms of stresses using the finite element method. Any commercial solver such as Nastran, Ansys, or Abaqus can be used for this purpose. The probability of failure-free operation (reliability) is calculated involving the variational series of loads and load-carrying ability. For this purpose, the formalized estimation method based on empirical distributions is used. Fundamentals of the latter are available in the monograph "Reliability in Engineering Design" written by Kapur K.C. and Lamberson L.R..

A primary function of the proposed method is to evaluate the probability of failure-free operation of constructions under stationary or quasi-stationary random loads.

Stanislav A. PIMENOV (Doctor of Technical Sciences, Branch of FSUE «RFNC-VNIIEF» «NIIIS named after Yu.Ye. Sedakov», Nizhny Novgorod, Russian Federation). E-mail: spimenov_m_fem@mail.ru

\section{REFERENCES}

1. Volkov V.M. (2011) Nadyozhnost' mashin i tonkostennykh konstruktsiy. Uchebnoe posobie [Reliability of machines and thin-walled constructions. Tutorial]. Nizhny Novgorod: NGTU.

2. Kapur K.C., Lamberson L.R. (2009) Reliability in Engineering Design. Wiley India Private Limited.

3. Pimenov S.A. (2010) Primenenie chislennykh metodov dlya otsenki nadezhnosti konstruktsiy [Application of numerical methods for evaluating the reliability of constructions]. Novye promyshlennye tekhnologii. 3. pp. 55-57.

4. Pimenov S.A., Palkin I.Yu. (2011) Primenenie rekursivnykh algoritmov pri otsenke nadezhnosti konstruktsiy [Application of recursive algorithms for evaluating the reliability of constructions]. Naukoyomkie tekhnologii - Science Intensive Technologies. 4. pp. 39-43.

5. Ostreykovskiy V.A. (2003) Teoriya nadyozhnosti [Reliability theory]. Moscow: Vysshaya shkola.

6. Probabilistic Design Methodology for Composite Aircraft Structures, National Technical Information Service (NTIS). Springfield, Virginia, 1999. Access mode: http://www.tc.faa. gov/its/worldpac/techrpt/ar99-2.pdf.

7. Zenkevich O.K. (1975) Metod konechnykh elementov v tekhnike [Finite element method in engineering]. Moscow: Mir.

8. NX Nastran Numerical Methods User's Guide. UGS, 2005. Access mode: http://docs.plm. automation.siemens.com/data_services/resources/nxnastran/10/help/en_US/custom/numerical /numerical.pdf.

9. ANSYS Mechanical APDL Basic Analysis Guide, Release 15.0. ANSYS Inc., 2013. Access mode: https://docplayer.net/45466427-Ansys-mechanical-apdl-basic-analysis-guide.html.

10. Abaqus Analysis User's Manual, Release 6.1. Dessault Systems, 2010. Access mode: https://www.academia.edu/24831145/Abaqus_Analysis_Users_Manual.

Received: February 17, 2020 